\title{
GM-CSF Enhances Macrophage Glycolytic Activity In Vitro and Improves Detection of Inflammation In Vivo
}

\author{
Parmanand Singh*1, Silvia González-Ramos*2, Marina Mojena ${ }^{2}$, César Eduardo Rosales-Mendoza ${ }^{2,3}$, Hamed Emami ${ }^{4}$, \\ Jeffrey Swanson ${ }^{4}$, Alex Morss ${ }^{4}$, Zahi A. Fayad ${ }^{5}$, James H.F. Rudd 6 , Jeffrey Gelfand ${ }^{7}$, Marta Paz-García ${ }^{2}$, \\ Paloma Martín-Sanz ${ }^{2,8}$, Lisardo Boscá $^{\dagger 2,8}$, and Ahmed Tawakol ${ }^{\dagger 4}$ \\ ${ }^{I}$ Cardiology Division, New York Presbyterian Hospital, Weill Cornell Medical College, New York, New York; ${ }^{2}$ Instituto de \\ Investigaciones Biomédicas "Alberto Sols," CSIC-UAM, Madrid, Spain; ${ }^{3}$ Departamento de Bioquímica y Medicina Molecular, \\ Universidad Autónoma de Nuevo León, Monterrey, Nuevo León, México; ${ }^{4}$ Cardiac MR PET CT Program, Cardiology Division, \\ Massachusetts General Hospital and Harvard Medical School, Boston, Massachusetts; ${ }^{5}$ Translational and Molecular Imaging \\ Institute, Icahn School of Medicine at Mount Sinai, New York, New York; ${ }^{6}$ Division of Cardiovascular Medicine, University of \\ Cambridge, Cambridge, United Kingdom; ${ }^{7}$ Division of Infectious Diseases, Department of Medicine, Massachusetts General Hospital \\ and Harvard Medical School, Boston, Massachusetts; and ${ }^{8}$ Centro de Investigación Biomédica en Red de Enfermedades Hepáticas y \\ Digestivas, Instituto de Salud Carlos III, Madrid, Spain
}

\begin{abstract}
${ }^{18} \mathrm{~F}-\mathrm{FDG}$ accumulates in glycolytically active tissues and is known to concentrate in tissues that are rich in activated macrophages. In this study, we tested the hypotheses that human granulocyte-macrophage colony-stimulating factor (GM-CSF), a clinically used cytokine, increases macrophage glycolysis and deoxyglucose uptake in vitro and acutely enhances ${ }^{18} \mathrm{~F}-\mathrm{FDG}$ uptake within inflamed tissues such as atherosclerotic plaques in vivo. Methods: In vitro experiments were conducted on human macrophages whereby inflammatory activation and uptake of radiolabeled 2-deoxyglucose was assessed before and after GM-CSF exposure. In vivo studies were performed on mice and New Zealand White rabbits to assess the effect of GM-CSF on ${ }^{18} \mathrm{~F}-\mathrm{FDG}$ uptake in normal versus inflamed arteries, using PET. Results: Incubation of human macrophages with GM-CSF resulted in increased glycolysis and increased 2-deoxyglucose uptake $(P<$ $0.05)$. This effect was attenuated by neutralizing antibodies against tumor necrosis factor- $\alpha$ or after silencing or inhibition of 6-phosphofructo-2-kinase. In vivo, in mice and in rabbits, intravenous GM-CSF administration resulted in a $70 \%$ and $73 \%$ increase $(P<0.01$ for both), respectively, in arterial ${ }^{18} \mathrm{~F}-\mathrm{FDG}$ uptake in atherosclerotic animals but not in nonatherosclerotic controls. Histopathologic analysis demonstrated a significant correlation between in vivo ${ }^{18} \mathrm{~F}-\mathrm{FDG}$ uptake and macrophage staining $(R=0.75, P<0.01)$. Conclusion: GM-CSF substantially augments glycolytic flux in vitro (via a mechanism dependent on ubiquitous type 6-phosphofructo-2-kinase and tumor necrosis factor- $a$ ) and increases ${ }^{18}$ F-FDG uptake within inflamed atheroma in vivo. These findings demonstrate that GM-CSF can be used to enhance detection of inflammation. Further studies should explore the role of GM-CSF stimulation to enhance the detection of inflammatory foci in other disease states.
\end{abstract}

\footnotetext{
Received Sep. 24, 2015; revision accepted Mar. 7, 2016.

For correspondence or reprints contact: Lisardo Boscá, Instituto de Investigaciones Biomédicas "Alberto Sols" (CSIC-UAM), Arturo Duperier 4, 28029 Madrid, Spain.

E-mail: Ibosca@iib.uam.es

${ }^{*}$ Contributed equally to this work.

tContributed equally to this work.

Published online Apr. 14, 2016.

COPYRIGHT (C) 2016 by the Society of Nuclear Medicine and Molecular Imaging, Inc.
}

Key Words: ${ }^{18} \mathrm{~F}-\mathrm{FDG}-\mathrm{PET}$; glycolysis; GM-CSF; inflammation; macrophage

J Nucl Med 2016; 57:1428-1435

DOI: 10.2967/jnumed.115.167387

\section{$\mathbf{P}$} ET, in combination with ${ }^{18} \mathrm{~F}-\mathrm{FDG}$, has been shown to be useful for the identification of inflamed tissues. Over the past few years, ${ }^{18}$ F-FDG PET/CT imaging has been put into increasing clinical use to evaluate syndromes such as fever of unknown origin, cardiac sarcoidosis $(1,2)$, prosthetic valve endocarditis (3), and infection of implanted devices (4). More recently, the use of ${ }^{18}$ F-FDG PET/CT as a research tool to characterize atherosclerotic cardiovascular disease has been increasing. However, more widespread use of ${ }^{18}$ F-FDG PET imaging of inflammatory foci has been limited, in part because of the relatively modest signal (5). Approaches to enhance ${ }^{18} \mathrm{~F}-\mathrm{FDG}$ localization to inflamed tissues could improve the clinical utility of ${ }^{18} \mathrm{~F}$-FDG PET imaging of inflammatory diseases.

Perhaps the most common chronic inflammatory disorder, atherosclerotic cardiovascular disease, remains the leading cause of mortality in the United States (6). Inflammation plays a pivotal role in atherogenesis, plaque progression, and thrombotic complications (7). In particular, the pathogenesis of atherosclerosis involves a myriad of immune mediators, with a well-accepted role for macrophages (8). A substantial body of cellular physiology literature has established that activated macrophages show upregulated glycolysis and hence avidly accumulate ${ }^{18} \mathrm{~F}$-FDG $(5,9)$. Key enzymatic mediators of macrophage activity include the glucose transporters, the upper part of glycolysis, and specifically the expression of the ubiquitous form of 6-phosphofructo-2-kinase (PFKFB3), which has been noted to be upregulated in stimulated macrophages (compared with the constitutively expressed liver type-6-phosphofructo-2-kinase [PFKFB1] isoenzyme, which is associated with lower rates of glycolysis) (9). Advances in ${ }^{18} \mathrm{~F}-\mathrm{FDG}$ PET imaging have led to its use for the quantification of vascular wall inflammatory activity. ${ }^{18} \mathrm{~F}$-FDG uptake has been shown to 
strongly correlate with arterial wall macrophage infiltration (10), systemic proinflammatory biomarkers (11), inflammatory cell gene expression (12), and increased risk for subsequent atherothrombotic events $(13,14)$.

Granulocyte-macrophage colony-stimulating factor (GM-CSF) is a Food and Drug Administration-approved medication used to stimulate the production of white blood cells and thus prevent neutropenia after chemotherapy. It affects a wide range of immune cells, including macrophages, neutrophils, and dendritic cells (15-18). The drug is well tolerated, even among individuals with atherosclerosis or those with recent atherothrombotic events $(19,20)$. Accordingly, we sought to evaluate whether GM-CSF could be used as an adjunctive agent to enhance the detection of inflamed foci. Specifically, we performed both in vitro and in vivo experiments to better understand the effect of GM-CSF on macrophage glycolytic flux and on ${ }^{18} \mathrm{~F}$-FDG uptake, which is dependent on glucose flux. In these studies, we tested the hypotheses that GM-CSF augments glycolytic flux in macrophages in vitro and enhances ${ }^{18} \mathrm{~F}-\mathrm{FDG}$ accumulation within inflammatory foci in vivo, thereby enhancing imaging sensitivity.

\section{MATERIALS AND METHODS}

\section{Cellular Experiments}

Chemicals. Reagents and antibodies were from Sigma-Aldrich, Roche, Invitrogen, R\&D Systems, Santa Cruz Biotech, or Merck-Millipore. $\left[\mathrm{U}-{ }^{14} \mathrm{C}\right]$-2-deoxyglucose $(9,250 \mathrm{kBq}[250 \mu \mathrm{Ci}] / \mathrm{mmol})$ was from New England Nuclear/Perkin Elmer. Serum and media were from BioWhittaker. Murine and human GM-CSFs were from PeproTech or Gentaur.

Preparation of Human Monocytes/Macrophages. PBMCs were isolated from the blood of healthy donors by centrifugation on FicollHypaque Plus (GE Biotech) following the manufacturer's protocol, and the CD14-enriched fraction was collected after binding to MACS-hCD14magnetic beads (Miltenyi Biotec). Cells were differentiated with human CSF-1 (20 ng/mL, PeproTech) for $5 \mathrm{~d}$ in RPMI1640 supplemented with antibiotics and $10 \%$ fetal calf serum. After this period, the cells were kept for $48 \mathrm{~h}$ in medium lacking CSF-1 and treated with the indicated stimuli. The purity of all cultures was verified by $\mathrm{CD}_{14}{ }^{+}$staining; on average, more than $95 \%$ of the cells were highly positive for this surface marker.

Tumor Necrosis Factor- $\alpha(T N F-\alpha)$ Neutralization. The cells were maintained in culture and, $1 \mathrm{~h}$ before GM-CSF challenge, were treated with a $20 \mathrm{ng} / \mathrm{mL}$ concentration of anti-TNF- $\alpha$ neutralizing antibody (R\&D Systems) as previously described (9), using a mouse IgG as control.

PFKFB3 Silencing in Macrophages. The cells were transfected overnight with lipofectamine and a mixture of 3 different Silencer Select predesigned silencer RNAs from Ambion/Invivogen, following the instructions of the supplier. Controls with scrambled (negative) RNAs were used to ensure the specificity of the silencing.

Measurement of Radiolabeled Deoxyglucose Accumulation. To evaluate 2-deoxyglucose uptake, the cells (6-cm dishes) were washed with warm Dulbecco modified Eagle medium lacking glucose and incubated with this medium containing $0.3 \mathrm{mM}$ glucose and $92.5 \mathrm{kBq}(2.5 \mu \mathrm{Ci})$ of [U-14 C]-2-deoxyglucose for $60-90 \mathrm{~s}$, following a previous protocol $(21)$. The reaction was stopped by rapid aspiration of the medium and addition of $1 \mathrm{~mL}$ of ice-cold Dulbecco modified Eagle medium containing $10 \mathrm{mM}$ glucose and $0.1 \mathrm{mM}$ phloretin. The cell layer was washed twice with icecold phosphate-buffered saline, and the cells were resuspended in $1 \mathrm{~mL}$ of $0.1 \mathrm{M} \mathrm{NaOH}$ in $0.1 \%$ sodium dodecyl sulfate. A linear incorporation of radioactivity was observed for at least $2 \mathrm{~min}$ by scintillation counting.

Preparation of Macrophage Extracts. The cell cultures were washed twice with ice-cold phosphate-buffered saline and homogenized in $0.2 \mathrm{~mL}$ of buffer containing $10 \mathrm{mM}$ Tris- $\mathrm{HCl}, \mathrm{pH} 7.5 ; 1 \mathrm{mM} \mathrm{MgCl}_{2} ; 1 \mathrm{mM}$ ethylene glycol tetraacetic acid; $10 \%$ glycerol; 0.5\% CHAPS buffer (3-[(3-cholamidopropyl)dimethylammonio]-1-propanesulfonate); $1 \mathrm{mM}$ $\beta$-mercaptoethanol; and $0.1 \mathrm{mM}$ phenylmethylsulfonyl fluoride and a protease inhibitor cocktail (Sigma). The extracts were stirred in a vortex mixer for $30 \mathrm{~min}$ at $4^{\circ} \mathrm{C}$ and centrifuged for $15 \mathrm{~min}$ at $13,000 \mathrm{~g}$. The supernatants were stored at $-20^{\circ} \mathrm{C}$. Protein levels were determined using the Bio-Rad detergent-compatible protein reagent. All steps were performed at $4^{\circ} \mathrm{C}$.

Western Blot Analysis. Samples of cell extracts containing equal amounts of protein $(30 \mu \mathrm{g}$ per lane) were boiled in $250 \mathrm{mM}$ Tris- $\mathrm{HCl}, \mathrm{pH}$ $6.8 ; 2 \%$ sodium dodecyl sulfate; $10 \%$ glycerol; and $2 \% \beta$-mercaptoethanol and size-separated in $10 \%-15 \%$ sodium dodecyl sulfate polyacrylamide gel electrophoresis. The gels were blotted onto a polyvinylidene fluoride membrane (GE Healthcare) and processed as recommended by the supplier of the antibodies against the following human antigens: signal transducer and activator of transcription 5 (STAT5), P-STAT5, hexokinase-1, hexokinase-2, PFK-1, PFKFB1, PFKFB3, glyceraldehyde 3phosphate dehydrogenase, and $\beta$-actin. The blots were developed by ECL protocol (GE Healthcare), and different exposition times were performed for each blot with a charge-coupled device camera in a luminescent image analyzer (Molecular Imager; BioRad) to ensure the linearity of the band intensities.

Measurement of TNF- $\alpha$, Lactate, and Fructose-2,6-Bisphosphate (Fru-2,6- $P_{2}$ ). TNF- $\alpha$ was measured in the cell culture medium using an ELISA kit (PeproTech). Lactate accumulation was also measured in the culture medium as previously described $(9,22)$. The intracellular concentration of Fru-2,6- $\mathrm{P}_{2}$ was determined in cell extracts treated with $0.1 \mathrm{~mL}$ of $\mathrm{NaOH}\left(50 \mathrm{mM}\right.$ at $\left.80^{\circ} \mathrm{C}\right)$ and heated at $80^{\circ} \mathrm{C}$ for $10 \mathrm{~min}$. The metabolite was measured after the activation of the pyrophosphatedependent 6-phosphofructo-1-kinase activity following a previous proto$\operatorname{col}(9,22)$.

Real-Time Quantitative Polymerase Chain Reaction. 3-mo-old Apoe $e^{-1-}$ mice were fed for 2 wk with a high-fat diet and intraperitoneally administered $37.5 \mu \mathrm{g} / \mathrm{kg}$ of GM-CSF on day 12 . On day 14 , they were sacrificed and the whole aorta was cleaned to remove the adventitial fat and connective tissue in the magnifying glass, always working over a sheet of ice. The aortas were transferred to RNAlater RNA stabilization reagent (QIAGEN) and then to dry ice. Aorta total RNA was isolated by homogenization in QIAzol (QIAGEN) by a Tissue Lyser LT (QIAGEN) and eluted in a volume of $15 \mu \mathrm{L}$ using MinElute columns (QIAGEN). RNA integrity was assessed by RNA 6000 Nano Kit (Agilent). A 250-ng quantity of RNA was retrotranscribed using a high-capacity complementary DNA reverse transcription kit (Life Technologies). Real-time polymerase chain reaction was conducted with SYBR Green (Life Technologies) in a fast real-time polymerase chain reaction system (Life Technologies). The real-time polymerase chain reaction assay was performed with $5 \mathrm{ng}$ of complementary DNA per well, and the thermocycling conditions were $95^{\circ} \mathrm{C}$ for $10 \mathrm{~min}$ and 40 cycles of $95^{\circ} \mathrm{C}$ for $15 \mathrm{~s}$ followed by $60^{\circ} \mathrm{C}$ for $1 \mathrm{~min}$. Calculations were made from measurement of triplicates of each sample. The relative amount of messenger RNA (mRNA) was calculated with the comparative $2^{-\Delta \Delta C t}$ method. Gene expression was normalized to 36b4. Primer sequences are available on request.

Statistical Analysis. The data shown are the mean \pm SD of 3-5 experiments. Statistical significance was estimated with the Student $t$ test for unpaired observations or ANOVA followed by the Bonferroni test when appropriate. Differences with values of $P$ less than 0.05 were considered statistically significant.

\section{Animal Model Studies}

Mouse Model. Animal care and experimental procedures were performed according to directive 2010/63/EU of the European Parliament, and the studies were approved by the Institutional Committee on Bioethics (authorization 28079-37A to the Instituto de Investigaciones Biomédicas). 
PFKFB3 In Vivo Silencing. A mixture of at least 3 different Silencer Select predesigned silencer RNAs for PFKFB3 was obtained from different sources (Ambion/InvivoGen, OriGene, or Sigma-Aldrich). The transfection mixture was prepared using Invivofectamine 2.0 (InvivoGen) and was administered intraperitoneally at $5 \mathrm{mg} / \mathrm{kg}$ per dose, following the instructions of the supplier. Administration of the corresponding scrambled (negative) RNAs was used to ensure the specificity of the silencing.

Atherogenesis in ApoE-Deficient Mice and ${ }^{18}$ F-FDG PET Image Analysis. Thirty male ApoE-deficient mice 3-4 mo old were fed a high-fat/high-cholesterol diet for $3 \mathrm{wk}$, and after anesthesia with isoflurane, ${ }^{18} \mathrm{~F}-\mathrm{FDG}$ (37 MBq/kg; $0.2 \mathrm{~mL}$ ) was administered intraperitoneally and the ${ }^{18} \mathrm{~F}$ emission was analyzed in a small-animal CT/ SPECT/PET system (Inveon; Siemens). The images were analyzed and quantified as previously described (9). Briefly, the first axial slice, representing the descending aorta (the first PET/CT slice clear of the aortic arch), and 5 consecutive slices at intervals of $3 \mathrm{~mm}$ were averaged to obtain the $\mathrm{SUV}_{\max }$. Measured background SUVs from the paraspinal muscles were used to obtain a corrected TBR. When PFKFB3 was silenced, the silencer RNAs were administered at days 3, 7, 10, and 12 after high-fat/high-cholesterol administration. A mixture of scrambled RNAs was used as control and administered at the same periods. GM-CSF (37.5 $\mu \mathrm{g} / \mathrm{kg}$ ) was intravenously administered on day 12 . The animals were processed on day 14 for images and biochemical analyses.

Rabbit Model. Nine male New Zealand White rabbits (Charles River Breeding Laboratories) were included in the study. Seven of the rabbits were initiated on a $0.3 \%$ cholesterol, $4.7 \%$ peanut oil hyperlipidemic diet for 6 mo to precipitate the development of atherosclerosis. One week after beginning the high-cholesterol diet, the animals were briefly anesthetized using ketamine and xylazine, and aortoiliofemoral denudation was performed by balloon catheter injury using a modified Baumgartner technique (23). Additionally, 2 control rabbits of similar size and identical origin were maintained on standard rabbit chow for $6 \mathrm{mo}$. No catheterization or other invasive procedure was performed on the control animals.

Rabbit PET/CT Imaging Protocol. After 6 mo of the prescribed diet, 8 atherosclerotic animals and 2 healthy controls underwent ${ }^{18} \mathrm{~F}$ FDG PET imaging. The animals were injected with a $37 \mathrm{MBq} / \mathrm{kg}$ dose of ${ }^{18} \mathrm{~F}-\mathrm{FDG}$, and PET images were obtained on a microPET P4 (Concorde MicroSystems) or similar system $3 \mathrm{~h}$ after ${ }^{18} \mathrm{~F}-\mathrm{FDG}$ administration to allow for maximum tracer uptake. The choice of time interval after ${ }^{18} \mathrm{~F}$-FDG injection was based on our previous work in animals (10). Images were obtained over $20 \mathrm{~min}$ and reconstructed using a filtered backprojection algorithm. The microPET P4 scanner is an animal PET tomograph with 32 planes over a $7.8-\mathrm{cm}$ axial extent, 19-cm transaxial field of view, and 22-cm animal port. Within $6 \mathrm{~d}$ of PET imaging, multidetector CT imaging was performed for anatomic coregistration.

GM-CSF Administration to Rabbits. After baseline imaging, 6 animals (4 atherosclerotic animals and 2 healthy controls) were injected with $100 \mu \mathrm{g}$ of sargramostim (yeast-derived recombinant human GMCSF; Berlex) daily for a total of $3 \mathrm{~d}$. Four atherosclerotic animals received saline injections at identical time points. One hour after the final injection on day 3, a $37 \mathrm{MBq} / \mathrm{kg}$ dose of ${ }^{18} \mathrm{~F}-\mathrm{FDG}$ was administered, and PET imaging was performed $3 \mathrm{~h}$ afterward. The biodistribution methods and results are provided in the supplemental data, available at http://jnm. snmjournals.org (Supplemental Fig. 1).

${ }^{18}$ F-FDG PET Image Analysis in Rabbits. The PET images were analyzed with masking of treatment allocation and temporal sequence. First, localization of aortic ${ }^{18} \mathrm{~F}$-FDG uptake was aided by coregistering the PET images with the multidetector CT images. The temporally masked baseline and follow-up PET image pairs were registered to their common multidetector CT images using a workstation that allows multimodal standard image fusion (REVEAL-MVS; Mirada Solutions)
(24). Thereafter, ${ }^{18} \mathrm{~F}-\mathrm{FDG}$ uptake could be compared for several stacked aortic segments over time. Aortic ${ }^{18} \mathrm{~F}$-FDG uptake was quantified by drawing a circular region of interest around the aorta in the axial view, in 4-mm increments (yielding a stack of 4-mm-thick aortic slices composing the imaged aorta). For each region of interest, the maximal SUV was recorded. The SUV is the decay-corrected tissue concentration of ${ }^{18} \mathrm{~F}$ FDG (in $\mathrm{kBq} / \mathrm{mL}$ ) divided by the injected dose per body weight. Background ${ }^{18} \mathrm{~F}$-FDG uptake was measured in the paraspinal muscles as an average of ten $20-\mathrm{mm}^{2}$ region-of-interest samples. $\mathrm{SUV}_{\max }$ was then background-corrected to derive a target-to-background ratio (TBR) for each aortic slice.

Histologic Analysis in Rabbits. After final imaging assessments, 3 atherosclerotic animals were sacrificed using an overdose of sodium pentobarbital. The aortas were excised, placed in $10 \%$ buffered formalin, and decalcified according to the standard protocol. The aortic samples were sectioned transversely at 5-mm intervals and stained with rabbit macrophage-specific monoclonal antibody RAM11 (Dako Corp.). RAM11 staining was calculated as a percentage of the stained area over the total cross-sectional area of the transversely sectioned aortic wall. Multiple segments from each rabbit abdominal aorta were obtained and compared against the corresponding PET axial slices. The averaged RAM11 staining for each 5-mm aortic segment was also obtained and compared with the mean TBR for the same segments. The association between anatomic histologic segments and corresponding PET images was based on distances from the renal arteries measured ex vivo compared with the coregistered multidetector CT images. Control animals were not sacrificed for histologic analysis because previous work has demonstrated that there is no inflammation (percentage rabbit antimacrophage antibodies [\%RAM11 staining]) within the aortic wall of control rabbits (24).

Statistical Analysis. Data were analyzed using SPSS, version 22 (IBM). Continuous parameters are reported as mean \pm SEM. Statistical analysis comparing the change in the TBR across atherosclerotic segments (before and after treatment with GM-CSF or saline) was performed using the Wilcoxon signed-rank test. The Spearman method was used to assess the correlation between atherosclerotic tissue uptake of ${ }^{18} \mathrm{~F}-\mathrm{FDG}$ after GM-CSF (measured by PET, as TBR) and the subsequent histopathologic assessment of inflammation in those same sections (\%RAM11 staining). A $P$ value of less than 0.05 was considered statistically significant.

\section{RESULTS}

\section{GM-CSF Augments Glycolytic Flux via Upregulation of PFKFB3}

In human macrophages, incubation with GM-CSF resulted in rapid phosphorylation of STAT5 in Y694 (Fig. 1A) and metabolic reprograming of macrophages (including modest increases in hexokinase-1 and hexokinase-2; Fig. 1B). Moreover, there was a substantial increase in PFKFB3, which was negligibly expressed before GM-CSF (Figs. 1B and 1C). The PFKFB3 isoform has a much higher net kinase activity than the constitutively expressed PFKFB1 isoenzyme. Thus, upregulation of PFKFB3 would be expected to produce substantially higher glycolytic flux. The specificity of this association was demonstrated using silencing RNA for PFKFB3, which resulted in attenuated induction of PFKFB3 (Fig. 1D), but not by the scrambled RNA sequence. Additionally, the selective PFKFB3 inhibitor 3-(3-pyridinyl)-1-(4-pyridinyl)-2-propen-1-one (3PO) did not alter the induction of this gene by GM-CSF (Fig. 1D).

In line with the increased expression of glycolytic enzymes, GM-CSF resulted in a substantial increase in glycolytic flux (measured as Fru-2-6- $\mathrm{P}_{2}$ concentration, a potent activator of glycolysis; Fig. 2A). This augmented glycolytic flux was attenuated 


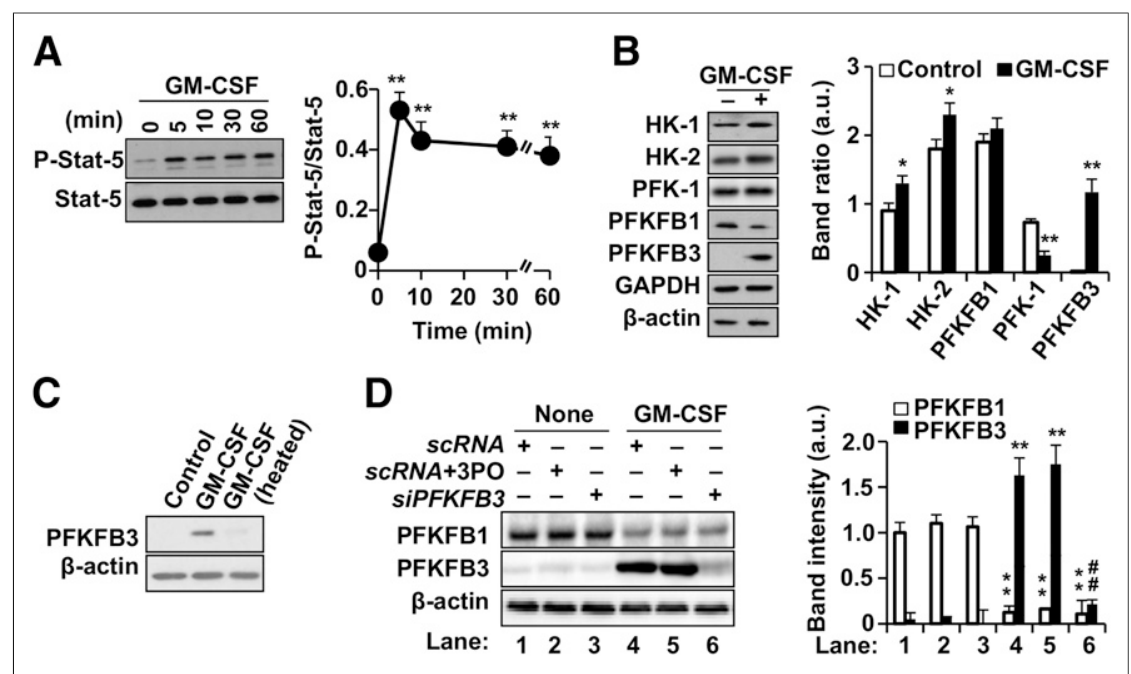

FIGURE 1. GM-CSF augments 2-deoxyglucose uptake and glycolytic flux in human macrophages via PFKFB3. (A and B) Human monocytes were differentiated to macrophages. Cells were exposed to human GM-CSF (10 ng/mL) for the specified time duration, and phosphorylation of STAT5 in Y694 (A) and levels of glycolytic enzymes (B) were determined. Addition of GM-CSF induced significant expression of hexokinase-1, hexokinase-2, and mainly PFKFB3. (C) Cells exposed to native GM-CSF demonstrated increased PFKFB3 expression compared with incubation with heat-inactivated $\mathrm{GM}-\mathrm{CSF}\left(10 \mathrm{~min}\right.$ at $\left.80^{\circ} \mathrm{C}\right)$. (D) $\mathrm{GM}-\mathrm{CSF}$-mediated increase in PFKFB3 expression (right) was decreased with addition of silencer to PFKFB3 but not by selective PFKFB3 inhibiter 3PO, demonstrating specificity of silencer. Cells were treated with mixture of siPFKFB3 for silencing PFKFB3 $18 \mathrm{~h}$ before challenge with GM-CSF. Results show mean \pm SD. ${ }^{\star} P<0.05$ vs. same condition in control (absence of GM-CSF or 0 h). ${ }^{\star \star} P<0.01$ vs. same condition in control (absence of GM-CSF or $0 \mathrm{~h}$ ). $\# \# P<0.01$ for $s c R N A$ vs. siPFKFB3 with or without 3PO. a.u. = arbitrary units; GAPDH = glyceraldehyde 3-phosphate dehydrogenase; $\mathrm{HK}=$ hexokinase; scRNA = scrambled RNA.

after inhibition of PFKFB3 by 3PO. In concert with the rise in Fru2-6- $\mathrm{P}_{2}$ concentration, GM-CSF also increased lactate accumulation in the medium (Fig. 2B).

Additionally, GM-CSF resulted in a selective (not achieved by heat-denatured GM-CSF) and modest increase in proinflammatory activation of the macrophages (as evidenced by macrophage TNF- $\alpha$ production; Fig. 2C). Furthermore, antagonism of TNF- $\alpha$ (using a neutralizing anti-TNF- $\alpha$ antibody) attenuated the glycolytic flux (measured as Fru-2-6- $\mathrm{P}_{2}$ concentration) after GM-CSF (Fig. 2D).

\section{GM-CSF Augments Deoxyglucose Accumulation Within Cultured Macrophages}

Incubation of macrophages with GM-CSF upregulated the incorporation of $\left[\mathrm{U}-{ }^{14} \mathrm{C}\right]$-2-deoxyglucose in a manner that paralleled its impact on glycolytic flux (Figs. 2E and 2F). A time-dependent increase in 2-deoxyglucose uptake was observed after GM-CSF challenge (Fig. 2E). The rate of 2-deoxyglucose uptake was significantly greater than in control cells, was increased as early as $2-4 \mathrm{~h}$ after GM-CSF exposure, and persisted, at a more pronounced rate of increase, at later time points $(P<0.01)$. Also, in parallel with the impact of GM-CSF on glycolysis, blocking of TNF- $\alpha$ or inhibition (3PO) or silencing (siPFKFB3) of PFKFB3 resulted in reduced 2-deoxyglucose uptake (Figs. 2E and 2F).

\section{GM-CSF Increases Atherosclerotic ${ }^{18}$ F-FDG Uptake In Vivo Without Altering Lesion Area}

The in vivo impact of GM-CSF on chronic atherosclerotic inflammation was investigated in 2 animal models (mouse and rabbit). In $A p o e^{-l-}$ mice fed for 2 wk on a high-fat diet, administration of GM-CSF (on day 12 of the diet) significantly enhanced the aortic ${ }^{18} \mathrm{~F}-\mathrm{FDG}$ uptake (TBR measured at $48 \mathrm{~h}$ after administration was increased by $70 \%$ relative to animals treated with vehicle; $P<0.01$ ), whereas aortic ${ }^{18} \mathrm{~F}-\mathrm{FDG}$ uptake did not increase in animals given both GM-CSF and silencer mRNA for PFKFB3 (Fig. 3A). When the lesion area was evaluated after oil red staining, the single-dose administration of GM-CSF did not influence the lipid accumulation (Fig. 3B). Moreover, the increased TBR was the result of a significant increase in target (arterial) activity, whereas the background activity did not significantly change with GM-CSF (Fig. 3C).

Further, to assess the impact of GM-CSF on potential infiltration of circulating monocytes into the aortic wall, the whole aorta from treated animals was analyzed using real-time quantitative polymerase chain reaction. As Figure 3D shows, the macrophagespecific gene, $L x r \alpha$, did not increase after GM-CSF administration when compared with specific endothelial and smooth muscle cell markers (i.e., F8, Sm22a, Myh11, and Colla1). Together, these results suggest a minimal contribution of GM-CSF, if any, to enhance the recruitment of monocytes to the lesion area, in agreement with previous data (9).

In atherosclerotic rabbits, GM-CSF administration was associated with an approximately $73 \%$ increase in ${ }^{18} \mathrm{~F}-\mathrm{FDG}$ uptake $(5.87 \pm 0.14$ vs. $10.09 \pm 0.40$ before vs. after GM-CSF, arterial TBR \pm SEM, $P<$ 0.01 ; Figs. $4 \mathrm{~A}$ and $4 \mathrm{~B})$. Atherosclerotic animals injected with saline showed no statistically significant change in TBR $(4.17 \pm 0.32$ vs. $4.08 \pm 0.20, P<0.01)$ over the same time period. Further, in healthy rabbits without atherosclerotic inflammation, GM-CSF administration was associated with a decrease in the arterial signal $(2.26 \pm 0.07$ vs. $1.47 \pm 0.17, P<0.01)$. In contrast, blood ${ }^{18} \mathrm{~F}$ FDG activity did not differ between rabbits given GMCSF and those given saline (supplemental data). Collectively, these data demonstrate that administration of GM-CSF augments ${ }^{18} \mathrm{~F}-\mathrm{FDG}$ uptake within the arterial wall in atherosclerotic but not in normal rabbits.

\section{Atherosclerotic ${ }^{18}$ F-FDG Uptake After GM-CSF Correlates with Macrophage Density}

Next, we tested the hypothesis that arterial ${ }^{18} \mathrm{~F}$-FDG uptake after GM-CSF correlates with histologic macrophage density. To do so, we compared ${ }^{18}$ F-FDG uptake in atherosclerotic rabbits with macrophage-specific \%RAM11 staining in histologic segments of aortas from sacrificed animals. We observed that ${ }^{18}$ F-FDG uptake significantly correlated with \%RAM11 staining $(R=0.76, P=0.002$; Fig. 5).

\section{DISCUSSION}

Here, we have shown that GM-CSF augments the glycolytic flux in vitro (via a mechanism that depends on PFKFB3) and 

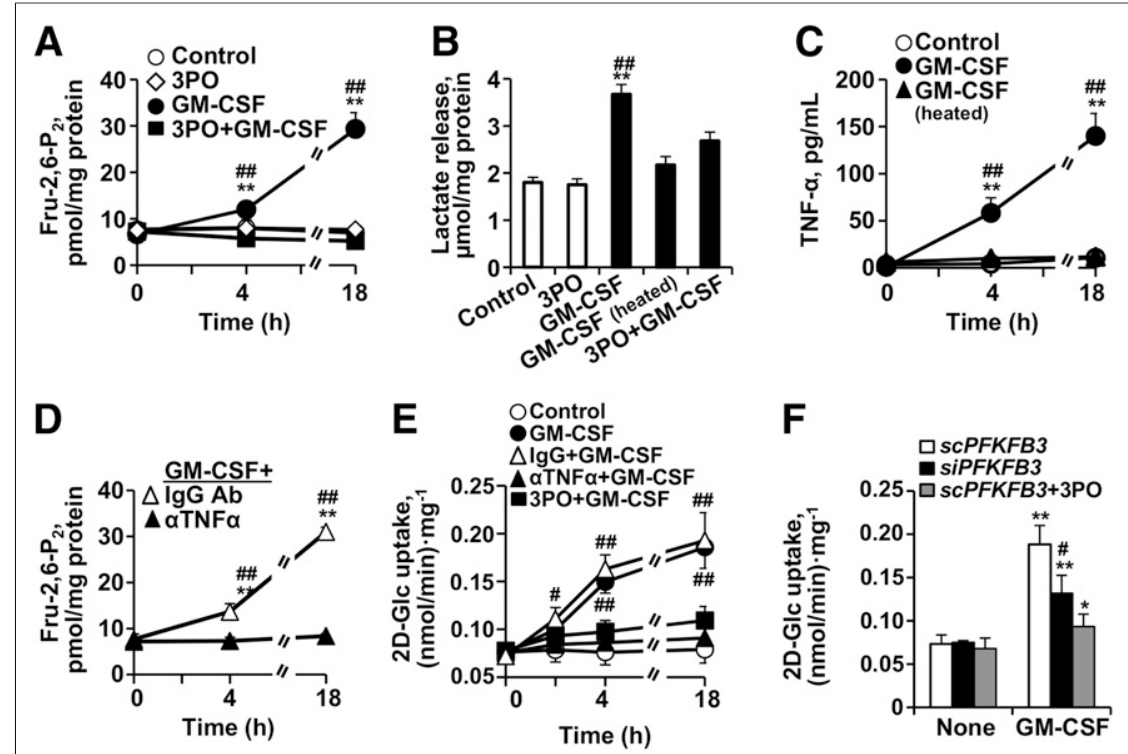

FIGURE 2. PFKFB3 mediates increase in 2-deoxyglucose uptake in human macrophages treated with GM-CSF. (A) Intracellular levels of Fru-2,6- $\mathrm{P}_{2}$ were determined at the indicated times with and without GM-CSF and in absence or presence of PFKFB3 inhibitor 3PO $(5 \mu \mathrm{M})$. GM-CSF augments Fru-2,6- $\mathrm{P}_{2}$ levels, which are attenuated in presence of $3 \mathrm{PO}$, demonstrating critical role of expression of PFKFB3 after GM-CSF treatment. (B) Lactate accumulation in culture medium was determined at $18 \mathrm{~h}$ with and without native or heat-inactivated GM-CSF and in absence or presence of 3PO $(5 \mu \mathrm{M})$. Lactate levels (by-product of glycolysis) increase with GM-CSF exposure and are dampened by 3PO. (C) TNF-a levels were determined in culture medium after challenge with native or heat-inactivated GM-CSF. (D) Intracellular levels of Fru-2,6- $\mathrm{P}_{2}$ were determined at the indicated times with GM-CSF and in presence of neutralizing anti-TNF-a antibody. GM-CSF augments Fru-2,6- $\mathrm{P}_{2}$ levels, which are attenuated in presence of neutralizing anti-TNF- $\alpha$ antibody. (E) 2-deoxyglucose uptake was determined at the indicated times with and without GM-CSF and in absence or presence of neutralizing anti-TNF-a antibody $(20 \mathrm{ng} / \mathrm{mL})$ or PFKFB3 inhibitor 3PO $(5 \mu \mathrm{M})$. 2-deoxyglucose significantly increases with addition of GM-CSF. This effect was diminished with neutralizing anti-TNF-a antibody and with 3PO. (F) To analyze contribution of PFKFB3 to enhancement of glycolytic flux elicited by GM-CSF, macrophages were transfected for $18 \mathrm{~h}$ with specific siRNA to silence PFKFB3 or with corresponding inactive RNA control (scRNA) and in absence or presence of $3 P O$, followed by activation for $18 \mathrm{~h}$ with GMCSF. 2-deoxyglucose uptake was significantly decreased after silencing PFKFB3 compared with corresponding control (scRNA) or inhibition with 3PO. Data are mean \pm SD. ${ }^{*} P<0.05$ vs. same condition in control (absence of GM-CSF or $0 \mathrm{~h}$ ). ${ }^{\star \star} P<0.01$ vs. same condition in control (absence of GM-CSF or $0 \mathrm{~h}$ ). ${ }^{\#} P<0.05$ for GM-CSF vs. heated GM-CSF, with 3PO plus GMCSF. $\# \#>0.01$ for GM-CSF vs. heated GM-CSF, with 3PO plus GM-CSF. IgG vs. anti-TNF-a antibody or sCRNA vs. SiPFKFB3 with or without 3PO. scRNA = scrambled RNA; siRNA = silencer RNA.

acutely increases ${ }^{18} \mathrm{~F}$-FDG uptake within inflamed tissues in vivo (in 2 animal atherosclerotic models). These data suggest that GMCSF, when administered as an adjunctive imaging agent, improves the sensitivity for detecting inflammatory foci. Further investigations to evaluate the clinical impact of GM-CSF in ${ }^{18} \mathrm{~F}$-FDG PET imaging are warranted.

GM-CSF is an endogenously circulating cytokine that is present at basal levels in serum. Recombinant human GM-CSF, a widely available and clinically used immunomodulatory agent, was first approved by the Food and Drug Administration in 1991 for the acceleration of myeloid recovery after autologous bone marrow transplantation. Since that time, the use of GM-CSF has expanded. GM-CSF acts to mobilize peripheral-blood progenitor cells, resulting in shorter durations of neutropenia in patients receiving induction chemotherapy for hematologic malignancies (25). Further, it is widely appreciated that GM-CSF increases the hematopoietic ${ }^{18} \mathrm{~F}$-FDG signal and that the heightened signal may persist for weeks after the last dose $(16,26-31)$. The findings of this study provide further understanding of the mechanisms underlying this observation.

Here, we show that GM-CSF acutely increases the expression of PFKFB3, resulting in increased glycolytic flux compared with the constitutively expressed PFKFB1, since PFKFB3 has a much higher net kinase activity, therefore increasing the intracellular levels of Fru-2,6- $\mathrm{P}_{2}$ and accelerating the upper part of glycolysis. Further, we found that the upregulation of PFKFB3 was necessary for the increased glycolytic flux seen with GM-CSF in both in vitro (human macrophages) and in vivo models (mice): blocking PFKFB3 (with silencer RNA specific for PFKB3 or inhibiting its activity using $3 \mathrm{PO}$ ) resulted in levels of glycolysis that were only slightly above baseline levels. Additionally, we observed that antagonism of TNF- $\alpha$ (using an antibody against TNF- $\alpha$ ) resulted in an attenuation of glycolytic flux after GMCSF. This finding is consistent with the prior observation that PFKFB3 activation results in augmented TNF- $\alpha$ production and that the TNF- $\alpha$ in turn leads to increased PFKB3 (via a mechanism that relies on HIF-1 $\alpha$ (9).

Moreover, we found that GM-CSF provided a roughly 3 -fold increase in glycolytic flux and a similar increase in macrophage 2-deoxyglucose uptake in vitro. Similarly, in the $A \mathrm{poe}^{-1-}$ mice and in the rabbit models of atherosclerosis, we observed an approximately $70 \%-73 \%$ increase in ${ }^{18}$ F-FDG uptake. Such increased ${ }^{18} \mathrm{~F}$-FDG uptake might provide improved detection of infectious foci.

The studies examining the effects of chronic GM-CSF administration on atherosclerosis have provided inconsistent findings (32). In one study, hyperlipidemic LDLR-/- mice with a GM-CSF deficiency exhibited a $20 \%-50 \%$ decrease in aortic lesion size, depending on the location of the lesions and the sex of the animals. In another, Apoe-/- mice treated with GMCSF chronically manifested an increased atherosclerotic-lesion area. However, in another study, Apoe-/- mice with a genetic deletion of GM-CSF demonstrated an increased atherosclerotic-lesion size (without changes in plasma cholesterol). It follows then, that both proatherogenic and antiatherogenic properties have been attributed to GM-CSF. Accordingly, the consequences of long-term GM-CSF administration have yet to be determined. However, clinical experience with short-term GM-CSF administration suggests no increased risk of cardiovascular complications (33).

\section{Clinical Implications}

The concept of transiently "stimulating" macrophages with GMCSF to improve the signal-to-noise ratio of inflammatory foci is analogous to the use of exercise stress testing to enhance the detection of myocardial ischemia. With electrocardiographic exercise treadmill 


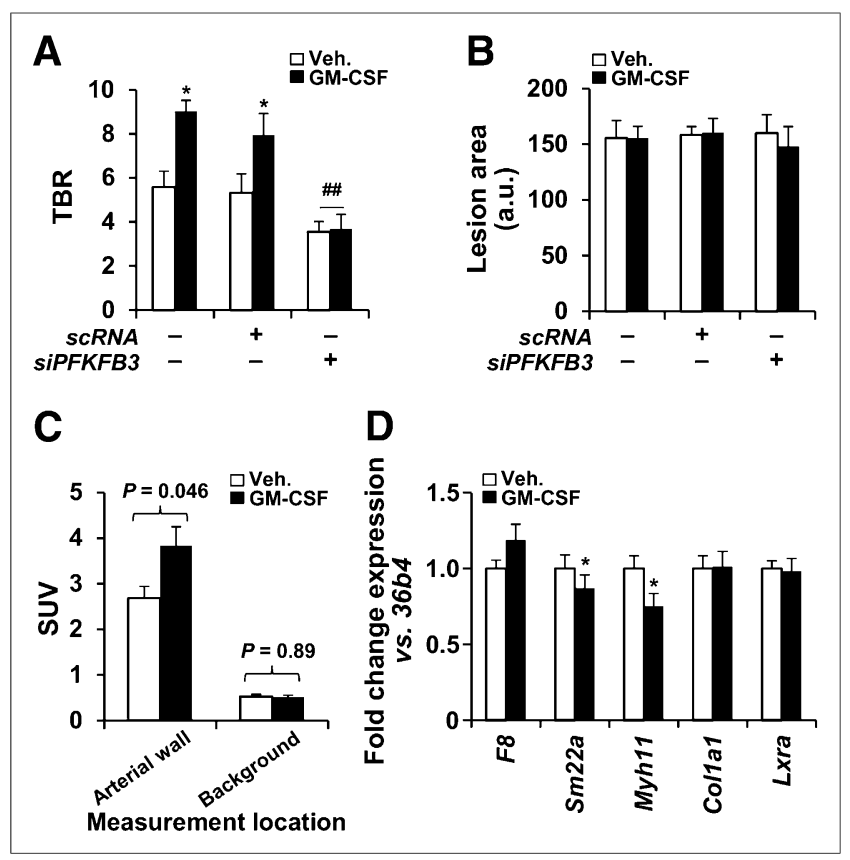

FIGURE 3. In vivo augmentation of ${ }^{18} \mathrm{~F}-\mathrm{FDG}$ uptake after GM-CSF administration in mice. (A) ${ }^{18} \mathrm{~F}-\mathrm{FDG}$ uptake in atherosclerotic mice $(n=$ 30). Animals received siPFKFB3 $(n=10)$ or $\operatorname{scRNA}(n=8)$ at days 3,7 , 10 , and 12 after high-fat/high-cholesterol administration. GM-CSF (37.5 $\mu \mathrm{g} / \mathrm{kg} ; n=15)$ or saline $(n=15)$ was intravenously administered on day 12 , and ${ }^{18} \mathrm{~F}-\mathrm{FDG}$ and PET analysis was performed on day 14 . (B) Aortas were stained with oil red and images evaluated with Image J. (C) Average target and background SUVs from A were compared ( $n=15$ animals for each group). Target SUVs were higher with than without GMCSF (3.38 \pm 0.46 vs. $2.70 \pm 0.26 ; P=0.046)$. Background SUVs were unchanged with vs. without GMCSF $(0.54 \pm 0.10$ vs. $0.55 \pm 0.10$; $P=0.53)$. (D) In parallel experiment, aortas from untreated $(n=4)$ or GM-CSF-treated animals $(n=4)$ as described in A were isolated and RNA extracted for analysis of the indicated genes representative of endothelial cells $(F 8)$, smooth muscle cells (Sm22a, Myh11, Col1a1), and macrophages (Lxra). Data are mean \pm SD. ${ }^{*} P<0.05$ vs. same condition in absence of GM-CSF. ${ }^{\# \# P}<0.01$ vs. same condition with scRNA. a.u. = arbitrary units; scRNA = scrambled RNA.

testing, the detection of occlusive coronary lesions (those that cause myocardial ischemia) is limited under resting conditions. However, when exercise is used to temporarily induce myocardial ischemia (leading to diagnostic changes on the electrocardiogram), a substantial boost in test sensitivity is yielded. At the same time, the induction of transient ischemia during exercise has been shown to be remarkably safe. Analogously, we propose further examination of the use of GM-CSF to transiently and safely provoke an inflammatory signal. This transient provocation may substantially improve the detection of inflammatory lesions, such as atherosclerotic or infectious foci.

Importantly, GM-CSF has a relatively safe track record, even when used for extended periods. The in vivo data presented within this study and from others provide some explanation of why GMCSF has a relatively modest proinflammatory profile compared with some other cytokines. We previously reported that lipopolysaccharide induces a 50- and 15-fold greater increase in TNF- $\alpha$ mRNA and protein production, respectively, compared with GMCSF (9). In that experiment as well, GM-CSF resulted in a 6-fold increase in Arg-1 mRNA, a prototypical marker of M2 polarization. In fact, the increase in Arg-1 mRNA exceeded the increase in
TNF- $\alpha$ mRNA, supporting the notion that GM-CSF provides a balanced increase in M1 as well as M2 polarization, yielding an intermediate polarization profile.

Moreover, the safety of GM-CSF has already been established in patients who have a preponderance of atherosclerotic risk factors, as well as in critically ill patients (33). In fact, GM-CSF may have benefits in chronic atherosclerosis (potentially related to augmentation of progenitor cell release) and is being examined as a therapy for chronic peripheral arterial disease (ClinicalTrials.gov identifier NCT01408901) $(34,35)$.

${ }^{18}$ F-FDG PET imaging has already become a critical tool in the localization of infections in the setting of fever of unknown origin $(36,37)$. Our findings offer justification to further explore the use of GM-CSF as an adjunctive agent for ${ }^{18} \mathrm{~F}$-FDG PET imaging of inflammation, in both acute and chronic inflammatory diseases (such as atherosclerosis and infections). In such cases, GM-CSF would be expected to transiently increase the ${ }^{18} \mathrm{~F}-\mathrm{FDG}$ signal within inflammatory cells and thus enhance the sensitivity, and possibly the specificity, of PET/CT imaging for detecting an inflammatory lesion.

\section{Use of GM-CSF as an Adjunctive Imaging Agent in Other Settings}

GM-CSF might also aid in the characterization of malignancies. ${ }^{18} \mathrm{~F}-\mathrm{FDG}$ PET/CT imaging has proven to be an invaluable tool in the workup of oncologic processes (38). The biologic basis of ${ }^{18} \mathrm{~F}$ FDG accumulation in malignant tumors results in part from the relatively high glycolytic rates found within malignant cells. However, tumor-associated macrophages also constitute an important fraction of oncologic tissues and provide an additional locus of intratumor ${ }^{18} \mathrm{~F}$-FDG accumulation (10). Occasionally, one cannot
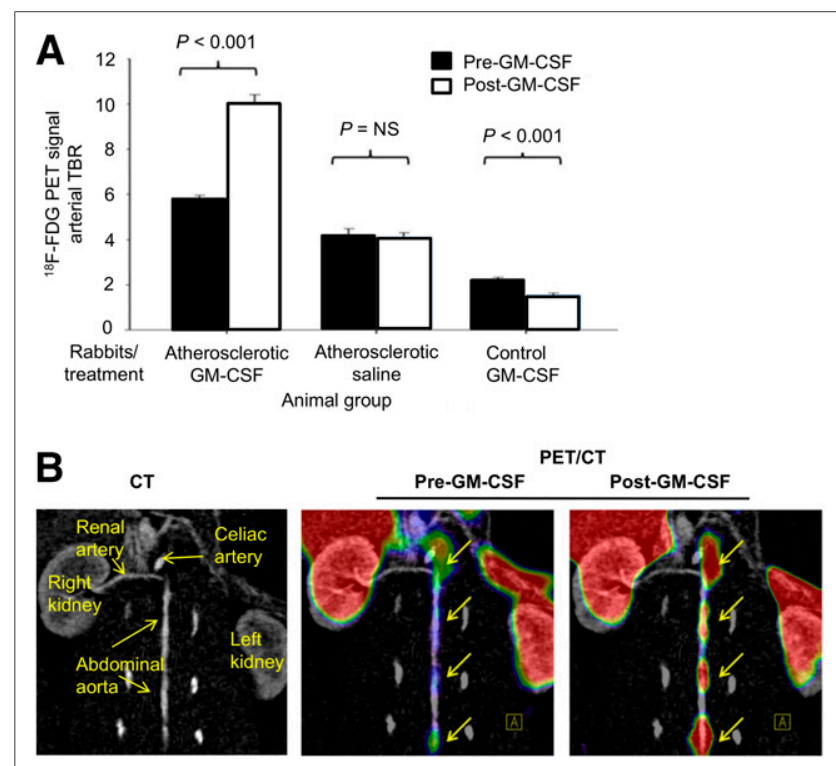

FIGURE 4. In vivo augmentation of ${ }^{18} \mathrm{~F}-\mathrm{FDG}$ uptake after GM-CSF administration in rabbits. (A) ${ }^{18} \mathrm{~F}-\mathrm{FDG}$ uptake in rabbits $(n=9)$ before and after challenge with cytokine GM-CSF or saline shows significant increase in ${ }^{18} \mathrm{~F}$-FDG uptake in atherosclerotic rabbits $(P<0.001) .{ }^{18} \mathrm{~F}$ FDG uptake is expressed as TBR compared with baseline imaging. (B) Representative image of ${ }^{18} \mathrm{~F}-\mathrm{FDG}$ signal enhancement demonstrating increased ${ }^{18} \mathrm{~F}-\mathrm{FDG}$ uptake in descending aorta before and after GM-CSF. 


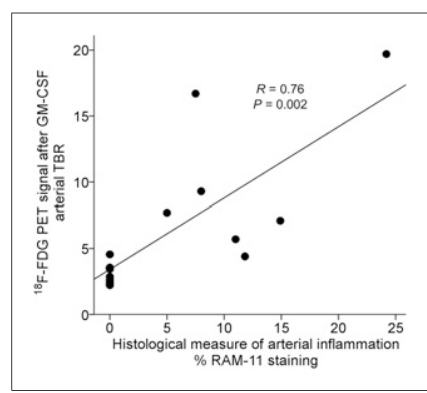

FIGURE 5. Relationship between arterial ${ }^{18} \mathrm{~F}-\mathrm{FDG}$ uptake (by PET/ CT) and histologic macrophage accumulation. Rabbits that had received GM-CSF (4 atherosclerotic and 2 control) were imaged using ${ }^{18} \mathrm{~F}-\mathrm{FDG}$ $\mathrm{PET} / \mathrm{CT}$. Arterial ${ }^{18} \mathrm{~F}-\mathrm{FDG}$ uptake was recorded as TBR. Subsequently, the animals were sacrificed and macrophage concentration within aortic wall was determined histologically (\%RAM11 staining). There was significant relationship between macrophage staining and TBR $(R=$ $0.76, P=0.002)$. nanoparticles. This is particularly facilitated by the fact that GM-CSF activates macrophages via mechanisms that promote both M2 (phagocytic) and mild M1 actions. With M2 activation comes an increase in uptake of nanoparticles such as superparamagnetic iron oxide (39). GM-CSF-enhanced imaging of superparamagnetic iron oxide and other nanoparticles should be further explored.

\section{Limitations}

This study is not without limitations. The optimal duration and dosing of GM-CSF was not examined in this study (only a 3-d GM-CSF regimen was examined). Thus, it is not clear from these data whether a single injection of GM-CSF would suffice to provide the desired boost in ${ }^{18} \mathrm{~F}$-FDG uptake. A multiday regimen would come at increased complexity, cost, and potential risks. Further, since only one imaging interval was studied, the optimal time point for ${ }^{18} \mathrm{~F}-\mathrm{FDG}$ PET/CT imaging after GM-CSF administration has not been determined. Future human studies are needed to assess the impact of a simplified GM-CSF regimen on ${ }^{18} \mathrm{~F}-\mathrm{FDG}$ PET/CT imaging.

\section{CONCLUSION}

GM-CSF augments macrophage glycolytic flux in vitro via a mechanism dependent on PFKFB3 and increases ${ }^{18}$ F-FDG uptake in vivo in animal atherosclerotic models. GM-CSF is an approved and clinically used medication with an established safety profile. Future larger studies should explore the potential of using GM$\mathrm{CSF}$ as an adjunctive imaging tool to improve detection of inflammation.

\section{DISCLOSURE}

The costs of publication of this article were defrayed in part by the payment of page charges. Therefore, and solely to indicate this fact, this article is hereby marked "advertisement" in accordance with 18 USC section 1734. James H.F. Rudd is in part supported by the NIHR Cambridge Biomedical Research Centre, the British
Heart Foundation, and HEFCE. Zahi A. Fayad is supported by NIH/NHLBI R01 HL071021, R01 HL078667, NIH/NBIB R01 EB009638, and the NIH/NHLBI Program of Excellence in Nanotechnology (PEN) Award, contract HHSN268201000045C. Parmanand Singh was supported by a grant from the NHLBI (5T32 HL076136) and is funded by the Marfan Foundation. Lisardo Boscá was supported by SAF2014-52492R, RTC2015-3741 from MINECO, and RD12/0042/0019. Centro de Investigación Biomédica en Red de Enfermedades Hepáticas y Digestivas is funded by the Instituto de Salud Carlos III and S2010/BMD-2378 from Comunidad de Madrid. Ahmed Tawakol is supported by NIH/ NHLBI R01 HL122177. No other potential conflict of interest relevant to this article was reported.

\section{REFERENCES}

1. Blankstein R, Osborne M, Naya M, et al. Cardiac positron emission tomography enhances prognostic assessments of patients with suspected cardiac sarcoidosis. J Am Coll Cardiol. 2014;63:329-336.

2. Osborne MT, Hulten EA, Singh A, et al. Reduction in ${ }^{18} \mathrm{~F}$-fluorodeoxyglucose uptake on serial cardiac positron emission tomography is associated with improved left ventricular ejection fraction in patients with cardiac sarcoidosis. J Nucl Cardiol. 2014;21:166-174.

3. Ricciardi A, Sordillo P, Ceccarelli L, et al. 18-fluoro-2-deoxyglucose positron emission tomography-computed tomography: an additional tool in the diagnosis of prosthetic valve endocarditis. Int J Infect Dis. 2014;28:219-224.

4. Sarrazin JF, Philippon F, Tessier M, et al. Usefulness of fluorine-18 positron emission tomography/computed tomography for identification of cardiovascular implantable electronic device infections. J Am Coll Cardiol. 2012;59:16161625 .

5. Tahara N, Mukherjee J, de Haas HJ, et al. 2-deoxy-2-[ $\left.{ }^{18} \mathrm{~F}\right]$ fluoro-D-mannose positron emission tomography imaging in atherosclerosis. Nat Med. 2014;20:215219.

6. Go AS, Mozaffarian D, Roger VL, et al. Heart disease and stroke statistics: 2014 update-a report from the American Heart Association. Circulation. 2014;129: e28-e292.

7. Libby P, Ridker PM, Maseri A. Inflammation and atherosclerosis. Circulation. 2002;105:1135-1143.

8. Libby P. Inflammation in atherosclerosis. Nature. 2002;420:868-874.

9. Tawakol A, Singh P, Mojena M, et al. HIF-1alpha and PFKFB3 mediate a tight relationship between proinflammatory activation and anaerobic metabolism in atherosclerotic macrophages. Arterioscler Thromb Vasc Biol. 2015;35:14631471 .

10. Tawakol A, Migrino RQ, Hoffmann U, et al. Noninvasive in vivo measurement of vascular inflammation with F-18 fluorodeoxyglucose positron emission tomography. J Nucl Cardiol. 2005;12:294-301.

11. Rudd JH, Myers KS, Bansilal S, et al. Relationships among regional arterial inflammation, calcification, risk factors, and biomarkers: a prospective fluorodeoxyglucose positron-emission tomography/computed tomography imaging study. Circ Cardiovasc Imaging. 2009;2:107-115.

12. Pedersen SF, Graebe M, Fisker Hag AM, Hojgaard L, Sillesen H, Kjaer A. Gene expression and ${ }^{18} \mathrm{FDG}$ uptake in atherosclerotic carotid plaques. Nucl Med Commun. 2010;31:423-429.

13. Figueroa AL, Abdelbaky A, Truong QA, et al. Measurement of arterial activity on routine FDG PET/CT images improves prediction of risk of future CV events. JACC Cardiovasc Imaging. 2013;6:1250-1259.

14. Rominger A, Saam T, Wolpers S, et al. ${ }^{18}$ F-FDG PET/CT identifies patients at risk for future vascular events in an otherwise asymptomatic cohort with neoplastic disease. J Nucl Med. 2009;50:1611-1620.

15. Fejer G, Wegner MD, Gyory I, et al. Nontransformed, GM-CSF-dependent macrophage lines are a unique model to study tissue macrophage functions. Proc Natl Acad Sci USA. 2013;110:E2191-E2198.

16. Fleetwood AJ, Lawrence T, Hamilton JA, Cook AD. Granulocyte-macrophage colony-stimulating factor (CSF) and macrophage CSF-dependent macrophage phenotypes display differences in cytokine profiles and transcription factor activities: implications for CSF blockade in inflammation. J Immunol. 2007;178:52455252.

17. Lacey DC, Achuthan A, Fleetwood AJ, et al. Defining GM-CSF- and macrophageCSF-dependent macrophage responses by in vitro models. J Immunol. 2012;188:5752-5765. 
18. Sugawara Y, Fisher SJ, Zasadny KR, Kison PV, Baker LH, Wahl RL. Preclinical and clinical studies of bone marrow uptake of fluorine-1-fluorodeoxyglucose with or without granulocyte colony-stimulating factor during chemotherapy. J Clin Oncol. 1998;16:173-180.

19. Miki T, Miura T, Nishino Y, et al. Granulocyte colony stimulating factor/macrophage colony stimulating factor improves postinfarct ventricular function by suppression of border zone remodelling in rats. Clin Exp Pharmacol Physiol. 2004;31:873-882.

20. Yano T, Miura T, Whittaker P, et al. Macrophage colony-stimulating factor treatment after myocardial infarction attenuates left ventricular dysfunction by accelerating infarct repair. J Am Coll Cardiol. 2006;47:626-634.

21. Bosca L, Rousseau GG, Hue L. Phorbol 12-myristate 13-acetate and insulin increase the concentration of fructose 2,6-bisphosphate and stimulate glycolysis in chicken embryo fibroblasts. Proc Natl Acad Sci USA. 1985;82:6440-6444.

22. Rodríguez-Prados JC, Traves PG, Cuenca J, et al. Substrate fate in activated macrophages: a comparison between innate, classic, and alternative activation. J Immunol. 2010;185:605-614.

23. Baumgartner HR. A new method for the induction of thrombi by controlled overdilatation of the vascular wall [in German]. Z Gesamte Exp Med. 1963;137:227247.

24. Tawakol A, Migrino RQ, Bashian GG, et al. In vivo ${ }^{18} \mathrm{~F}$-fluorodeoxyglucose positron emission tomography imaging provides a noninvasive measure of carotid plaque inflammation in patients. J Am Coll Cardiol. 2006;48:18181824 .

25. Arnberg H, Letocha H, Nou F, Westlin JF, Nilsson S. GM-CSF in chemotherapyinduced febrile neutropenia: a double-blind randomized study. Anticancer Res. 1998;18:1255-1260.

26. Campbell IK, van Nieuwenhuijze A, Segura E, et al. Differentiation of inflammatory dendritic cells is mediated by NF-kappaB1-dependent GM-CSF production in CD4 T cells. J Immunol. 2011;186:5468-5477.

27. Dhar-Mascareno M, Chen J, Zhang RH, Carcamo JM, Golde DW. Granulocytemacrophage colony-stimulating factor signals for increased glucose transport via phosphatidylinositol 3-kinase- and hydrogen peroxide-dependent mechanisms. J Biol Chem. 2003;278:11107-11114.

28. Khameneh HJ, Isa SA, Min L, Nih FW, Ruedl C. GM-CSF signalling boosts dramatically IL-1 production. PLoS One. 2011;6:e23025
29. Kim HJ, Oh JS, An SS, et al. Hypoxia-specific GM-CSF-overexpressing neural stem cells improve graft survival and functional recovery in spinal cord injury. Gene Ther. 2012;19:513-521.

30. Schneider UC, Schilling L, Schroeck H, Nebe CT, Vajkoczy P, Woitzik J. Granulocytemacrophage colony-stimulating factor-induced vessel growth restores cerebral blood supply after bilateral carotid artery occlusion. Stroke. 2007;38:1320-1328.

31. Spielholz C, Heaney ML, Morrison ME, Houghton AN, Vera JC, Golde DW. Granulocyte-macrophage colony-stimulating factor signals for increased glucose uptake in human melanoma cells. Blood. 1995;85:973-980.

32. Kleemann R, Zadelaar S, Kooistra T. Cytokines and atherosclerosis: a comprehensive review of studies in mice. Cardiovasc Res. 2008;79:360-376.

33. Abdel-Latif A, Bolli R, Zuba-Surma EK, Tleyjeh IM, Hornung CA, Dawn B. Granulocyte colony-stimulating factor therapy for cardiac repair after acute myocardial infarction: a systematic review and meta-analysis of randomized controlled trials. Am Heart J. 2008;156:216-226 e219.

34. Domanchuk K, Ferrucci L, Guralnik JM, et al. Progenitor cell release plus exercise to improve functional performance in peripheral artery disease: the PROPEL Study. Contemp Clin Trials. 2013;36:502-509.

35. Subramaniyam V, Waller EK, Murrow JR, et al. Bone marrow mobilization with granulocyte macrophage colony-stimulating factor improves endothelial dysfunction and exercise capacity in patients with peripheral arterial disease. Am Heart J. 2009;158:53-60.e1.

36. Blockmans D, Knockaert D, Maes A, et al. Clinical value of $\left[{ }^{18} \mathrm{~F}\right]$ fluoro-deoxyglucose positron emission tomography for patients with fever of unknown origin. Clin Infect Dis. 2001;32:191-196.

37. Bleeker-Rovers CP, de Kleijn EM, Corstens FH, van der Meer JW, Oyen WJ. Clinical value of FDG PET in patients with fever of unknown origin and patients suspected of focal infection or inflammation. Eur J Nucl Med Mol Imaging. 2004;31:29-37.

38. Lardinois D, Weder W, Hany TF, et al. Staging of non-small-cell lung cancer with integrated positron-emission tomography and computed tomography. N Engl J Med. 2003;348:2500-2507.

39. Satomi T, Ogawa M, Mori I, et al. Comparison of contrast agents for atherosclerosis imaging using cultured macrophages: FDG versus ultrasmall superparamagnetic iron oxide. J Nucl Med. 2013;54:999-1004. 